\title{
Running Faster Together: Huge Speed up of Thermal Ratchets due to Hydrodynamic Coupling
}

\author{
Paolo Malgaretti, ${ }^{1, *}$ Ignacio Pagonabarraga, ${ }^{1}$ and Daan Frenkel ${ }^{2}$ \\ ${ }^{1}$ Department de Fisica Fonamental, Universitat de Barcelona, Carrer Marti i Franques 1, 08028 Barcelona, Spain \\ ${ }^{2}$ Department of Chemistry, University of Cambridge, Lensfield Road, Cambridge CB2 1EW, United Kingdom \\ (Received 4 December 2011; revised manuscript received 5 September 2012; published 17 October 2012)
}

\begin{abstract}
We present simulations that reveal a surprisingly large effect of hydrodynamic coupling on the speed of thermal ratchet motors. The model that we use considers particles performing thermal ratchet motion in a hydrodynamic solvent. Using particle-based, mesoscopic simulations that maintain local momentum conservation, we analyze quantitatively how the coupling to the surrounding fluid affects ratchet motion. We find that coupling can increase the mean velocity of the moving particles by almost 2 orders of magnitude, precisely because ratchet motion has both a diffusive and a deterministic component. The resulting coupling also leads to the formation of aggregates at longer times. The correlated motion that we describe increases the efficiency of motor-delivered cargo transport and we speculate that the mechanism that we have uncovered may play a key role in speeding up molecular motor-driven intracellular transport.
\end{abstract}

PACS numbers: 87.16.Nn, 05.40.- a, 47.63.-b, 87.16.Wd

The motion of particles in a fluid is affected by the hydrodynamic interaction mediated by the embedding medium [1]. The dynamic coupling of small suspended particles affects, for instance, the average velocity of particles driven by a constant force [1] and the correlation spectrum of pairs of freely diffusing particles [2,3]. All existing studies show that hydrodynamic coupling can increase the speed at which particles move, but the resulting speed-ups are typically quite modest [4,5].

In this Letter we show that hydrodynamic interactions (HI) can cause a very large speedup of particles that move asynchronously by a thermal ratchet mechanism: hydrodynamic coupling increases the speed of such motors by up to 2 orders of magnitude compared to the velocity of isolated particles. Physical realizations of such ratchet motors, to which we will refer generically as steppers, can be created in colloidal systems [6] and may be found in molecular motors [7] that move along polar biofilaments, such as microtubules or actin. Hence, the effect of hydrodynamic coupling on stepping particles is likely to be relevant for the understanding of the physical mechanisms underlying intracellular transport processes such as cytoplasmic streaming [8], axonal transport $[9,10]$ and membrane-embedded cargo pulling [11].

In order to study the behavior of many steppers moving along the same filament, we employ a simple model that accounts both for the essential features of steppers and for the time-dependent hydrodynamics of the embedding fluid. The moving particles are described using the two-state ratchet model [7], a standard, simplified model that accounts for the mechanochemical coupling underlying molecular motor mechanics. In this ratchet model, the stepper can be in two different internal states: in state 1 particles displace under the action of a potential, $V(x)$, of period $l$, which depends on the position, $x$, along the filament axis and that we consider piecewise linear for simplicity. In state 2, particles undergo thermal diffusion along the filament. Steppers can switch from state 1 to state 2 with probability $p_{12}$ in a region of width $\epsilon$ around the potential minimum. The time spent diffusing in state 2 is determined by the homogeneous probability $p_{21}$ to jump back to state 1 . For energy consuming particles, the ratio $p_{12} / p_{21}$ differs from its thermal equilibrium ratio, thus breaking detailed balance and leading to rectified motion along the filament (see Fig. 1) [12].

To account efficiently for hydrodynamics that capture the geometrical confinement in which steppers displace, the fluid surrounding the filaments and moving particles is

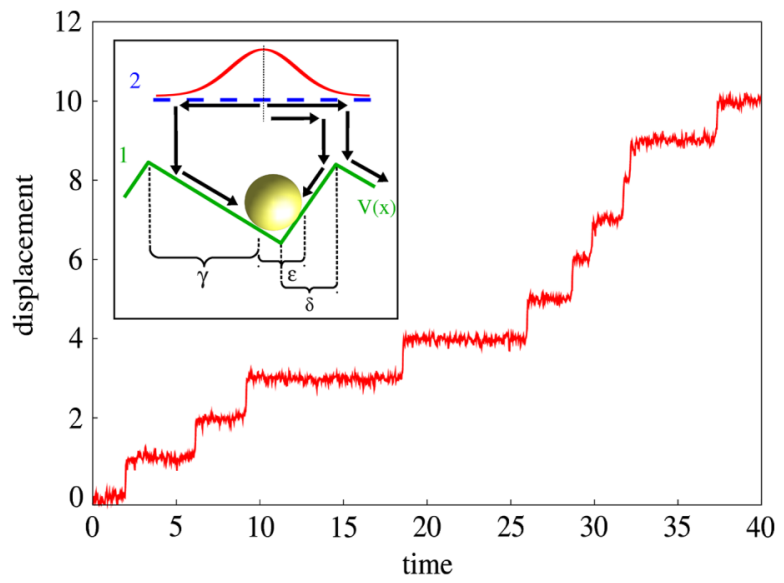

FIG. 1 (color online). Typical trajectory generated by a twostate ratchet model [7] for a single stepper. Inset: free-energy landscape. In the $\gamma$ region of state 1 (power stroke phase) the particle experiences the potential $V_{1}$. In region $\epsilon$ the particle can jump to the state 2 with a rate $p_{12}$. In state 2 (diffusive phase) the particle diffuses and can revert to state 1 with a rate $p_{21}$. Parameter values used in the simulations: $\gamma=0.75, \varepsilon=0.01$, $\delta=0.2, \Delta V_{1}=200$ (maximum energy difference); lengths in units of the ratchet period $l$ and energy in units of $k_{B} T$. 
modeled as an ideal dissipative fluid where point particles closer than a prescribed cutoff distance, $r_{c}$, interact through the Lowe-Andersen thermostat [13]. Fluid particles within $r_{c}$ from the filament surface or moving particles exchange momentum using the same local thermostat. The model thus enforces local equilibrium and linear and angular momentum conservation, yet it does not change the freeenergy landscape of the ratchet model. The spatial extent of moving particles is taken into account modeling them as hard, spherical particles with radius $a$. We have studied the collective motion of steppers that move at a fixed distance $r_{0}$ from the surface of a straight, fixed, filament of cross section $\pi r_{0}^{2}$. The filament is aligned parallel to one of the edges of our simulation box, of length $L \sim 100 l$ and square section with edge $L_{x}=L_{y}=24 l$ and periodic boundary conditions are applied (Similar results have been obtained for cylindrical confinement). For simplicity, moving particles never detach from the filament (i.e., they are infinitely processive according to the classification of molecular motors). Starting from a particle random distribution along the filament, we monitor the stepper average velocity $v$, and its dispersion on time scales during which moving particles can cross the periodic unit box $\left(t \leq L / v_{0}\right)$, being $v_{0}$ the average velocity of an isolated particle.

Before turning to HI, we first briefly consider the role of excluded-volume interactions (EV) among steppers. The effect of EV on $v$ depends on the ratio $R=a / l$. For biological molecular motors $R$ is of order 1, but in artificial ratchets it can be tuned by controlling the range of particleparticle interactions [14]. Figure 2 shows the variation of $v$ with the moving particle filament coverage. The results in Fig. 2 were obtained when particles move along a single track (prescribed by a confining angular potential) but this assumption is not essential: similar results are obtained if particles are allowed to spread on the filament surface. The mean velocity is sensitive to the precise value of the ratio $R$. A monotonic increase of $v$ with filament coverage $\left(\phi_{1 \mathrm{D}}=2 a N / L\right.$, with $N$ the number of particles on the filament) is observed for incommensurate moving particles, opposed to what is observed for passive diffusion.

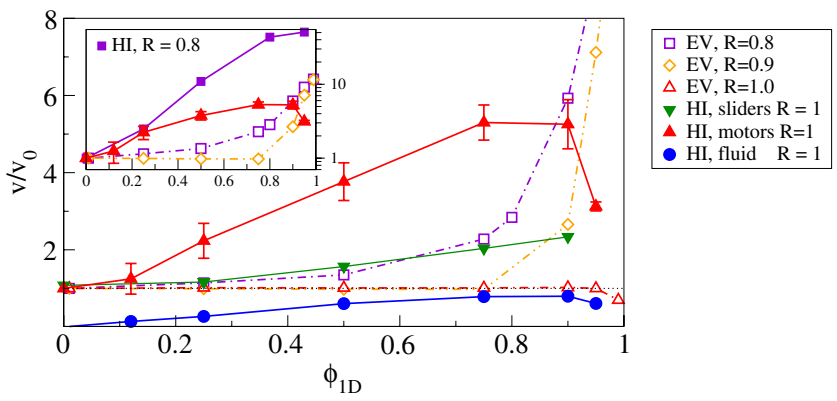

FIG. 2 (color online). Average velocity of steppers as a function of particle filament coverage, $\phi_{1 \mathrm{D}}$. For comparison the corresponding results for "sliders" (see text) are also shown: open and filled down-triangles. Inset: comparison between $R=1$ (triangles) and $R=0.8$ (squares) steppers including $\mathrm{HI}$.
This behavior can be understood because when the range of particle-particle repulsion is not commensurate with the ratchet period, a stepper in the diffusive state may be pushed toward the ratchet maximum (i.e., to the right into $\delta$ in the inset of Fig. 1) by a neighbor staying on its left (in region $\gamma$ ). Hence, steppers speed up due to the decrease in the time it takes them to move to the next ratchet minimum. When the particle size is commensurate with the ratchet period, $R=1, v$ depends only weakly on filament coverage, except at very high concentrations where manyparticle ratchet motion becomes less efficient than isolated particle motion. The motion of tightly elastically coupled motors also displays an analogous dependence on the commensurability of motor separation with the ratchet period $[15,16]$.

To focus on the effect of HI, we consider independent, processive steppers of size $R=1$, where EV are unimportant. Fig. 2 shows the dependence of $v$ for particles walking along a filament, as a function of the fractional filament particle coverage, $\phi_{1 \mathrm{D}}$. For $\bar{\phi}_{1 \mathrm{D}} \leq 0.1$, the average particle velocity does not depend on concentration but at higher concentrations, we observe a fairly linear dependence of $v$ on $\phi_{1 \mathrm{D}}$. The velocity increase is significantly larger than that observed for hydrodynamically coupled particles that move on a surface under the influence of a constant external force that has been chosen such that it reproduces the average velocity of isolated particles (referred to as "sliders" in Fig. 2) [5]. The key difference between sliders and the ratchet motion characteristic of steppers is that sliders move smoothly, rather than in bursts. A simple mean-field argument can be used to estimate the particle density at which hydrodynamic speed-up becomes significant. The average fraction $\rho p_{\downarrow}$ of bound particles which move under the influence of the ratchet force, $f$, (state 1) induces a mean drift velocity $v \simeq 2 \frac{f}{6 \pi \eta a} \rho p_{\downarrow} \frac{l-\delta}{l} \int_{2 R}^{L / 2} d r \frac{3 a}{2 r}$ on the diffusing steppers (state 2 ) over the characteristic time $\Delta t \simeq \frac{1}{2} \frac{6 \pi \eta a}{f(l-\delta)}$ in which bound particles displace along the filament. Hydrodynamic correlated motion plays a role when the mean displacement felt by the diffusing particles due to the ratcheted ones allows them to surmount the characteristic potential barrier, i.e., $v \Delta t \geq \delta$. Hence, hydrodynamic speed-up becomes significant for $\phi_{1 \mathrm{D}} \geq$ $\bar{\phi}_{1 \mathrm{D}} \equiv 2 \delta /(l-d)^{2} \ln L / 4 a$, which corresponds to $\bar{\phi}_{1 \mathrm{D}} \simeq$ 0.1 for $\delta=0.2, R=1, \tilde{L}=100$ and $p_{\downarrow} \sim 1$.

This hydrodynamic coupling, independent on the direct forces steppers exert on each other, is qualitatively different from the collective motion of particles interacting through short range forces where the possibility to enhance collectively their velocity depends on the degree of attraction and its commensurability with the potential ratchet $[4,17]$. When $\phi_{1 \mathrm{D}}$ approaches one, EV lead to a substantial decrease of the mean particle velocity. Figure 2 also shows that collective motion induces a net average flow of the fluid in which the filament and particles are embedded. Hence, active ratchet motion favors also the transport of 

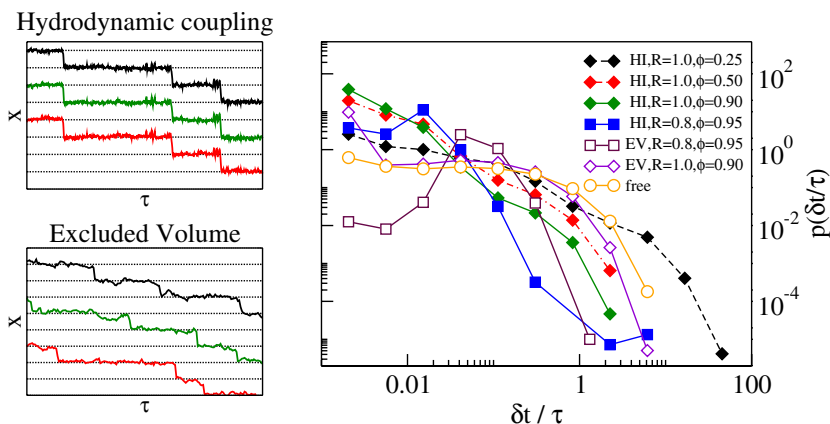

FIG. 3 (color online). Left: Correlated (uncorrelated) motion of three adjacent particles with HI (EV). Right: probability distribution of the dwell time, $\delta t$, between the consecutive stepping of neighbor steppers.

suspended, passive particles. This transport scenario is likely to be relevant for cargo motion in elongated geometries found in biological systems such as neurons and in plant cells. If the particle size is not commensurate with the ratchet period, $R<1$, the average stepper velocity is further enhanced, as shown in the inset of Fig. 2.

The probability distribution function of dwell times, $\delta t$, between subsequent stepping events of nearby particles provides insight into the mechanism of hydrodynamic velocity enhancement. As shown in Fig. 3, when hydrodynamic coupling is taken into account a sharp peak for small $\delta t$ appears indicating that HI favors the correlated motion of subsequent particles. When a particle steps, it generates a transient flow that pushes (pulls) the particles that are in front (behind) it, thus facilitating their crossing of the ratchet barrier. A similar speed-up has been described for colloids moving in a saw-tooth potential, under the influence of a constant, external force [15]. Such behavior is not observed for EV interactions that, for commensurate motors $(R=1)$ show a behavior very close to the, reference, behavior of free motors (nor HI nor EV interaction), while for smaller $R$, the short-ranged interactions between moving particles hinder their relative displacements, which favors shorter dwell times. The left panels of Fig. 3 display typical trajectories of three nearby steppers; in the presence of HI (top), jumps are strongly correlated, while no correlated jumps are observed in the absence of HI (bottom).

Thermal ratchet motors are not necessarily restricted to move along a single track. For example, in biological systems microtubules indeed are composed by a number of polarized tracks arranged in a cylindrical filament. To mimic the latter scenario we allowed particles to displace freely on the filament surface while subject to the same filament interaction, $V(x)$. An even stronger increase in $v$ is observed now as a function of the surface particle coverage, $\phi_{2 D}=N a^{2} /(2 r L)$, as shown in Fig. 4 . The more homogeneous filament coverage with respect to single track motion leads to average particle velocities that are 1 order of magnitude larger and it results in a stronger

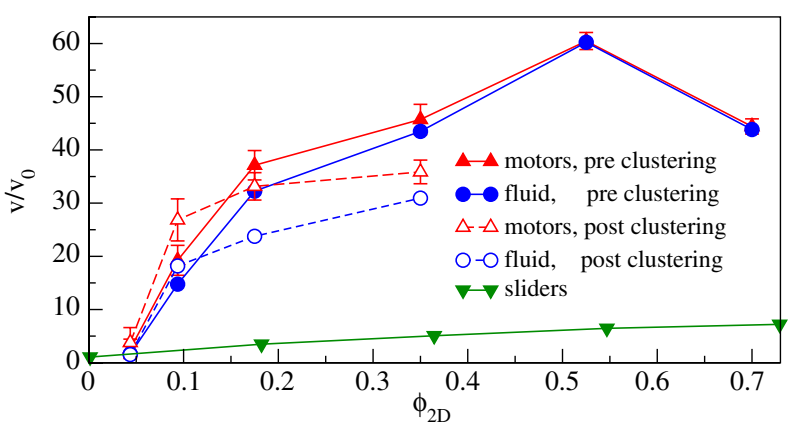

FIG. 4 (color online). HI-induced clustering of steppers moving on the cylindrical surface of the filament. Closed symbols show the average velocity of steppers (triangles) and fluid (circles) before clustering. The corresponding open symbols show the velocity after clustering. "Sliders" (downward triangles) clustering does not affect the mean velocity.

coupling to the surrounding fluid; the average flow velocity is comparable to that of the steppers. This implies that collective active motion can induce efficient passive intracellular transport. As in the single track case, there is a characteristic threshold coverage, $\bar{\phi}_{2 D}$, above which hydrodynamic speed up of $v$ is observed.

The fact that the mean particle velocity depends on the stepper concentration leads to "bunching." A group of moving particles with a higher than average concentration will move faster than the rest, thus leading to the buildup of larger clusters. This clustering results in heterogeneous filament coverage. We have analyzed cluster formation of stepper aggregates on long time scales, $t \gg L / v_{0}$. Using a robust distance criterion to identify clusters [18], we find that at intermediate and high filament coverage particles aggregate into a single cluster that survives for the rest of
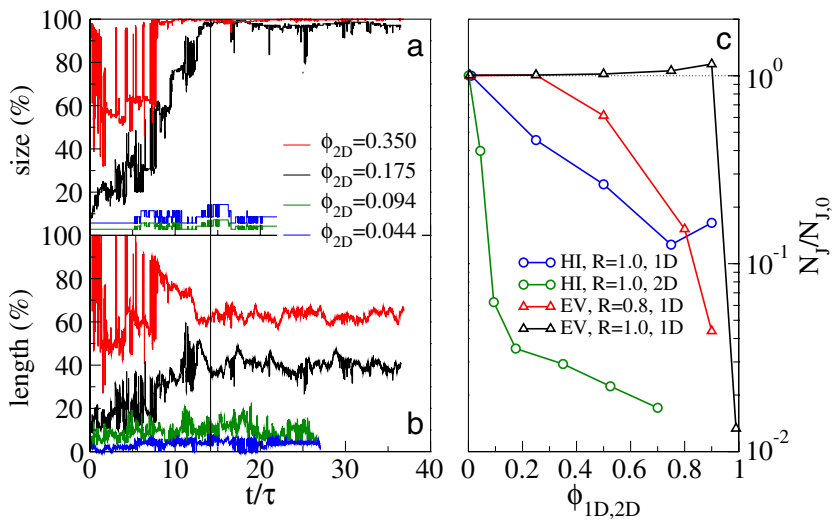

FIG. 5 (color online). Left: Fraction of steppers belonging to the largest cluster, as a function of time. The time unit $\tau \equiv L / v_{0}$. Right: Average number of stepper power strokes to displace one ratchet period. The energy consumption is proportional to the number of power strokes. Up (Down) triangles stands for steppers interacting through $\mathrm{EV}$ and $R=0.8(R=1)$ while closed (open) circles stand for steppers interacting through $\mathrm{HI}$ for single track (free diffusion on the filament surface) motion. 
the simulation up to twice the time needed to form the aggregate [see panel (a) of Fig. 5]. At long times the average particle speed and the coupling with the embedding fluid decrease slightly, as shown in Fig. 4, underlining that the heterogeneous coverage of the filament jointly with an increased effective filament coverage caused by clustering affect the particle collective motion. As the mean filament coverage increases, the aggregate percolates along the filament. At low filament coverages, $\phi_{2 D}<0.15$, we do not observe large aggregates. Rather, steppers organize into small clusters that form and dissolve. However, we cannot rule out that clusters develop at longer times.

Clearly, the hydrodynamic speed-up of moving particle groups increases transport efficiency. Assuming the transition rate between the two internal states of a particle is independent of filament coverage, the energy consumption of noninteracting steppers is constant. A good measure of particle displacement efficiency is then the average number of cycles, $N_{J}$, between the two internal energy states required to make a particle move one period. Panel (b) of Fig. 5 shows that $N_{J}$ decreases sharply when the steppers speed-up due to HI. For EV the decrease in $N_{J}$ is much more gradual and it is essentially absent if the size of the particle is commensurate with $V(x)$. The sharp decrease of $N_{J}$ with coverage illustrates that, as a result of hydrodynamic coupling, many particles "surf along" on the flow field generated by the power stroke of a single stepper.

We have shown that dynamic interactions among weakly coupled steppers strongly affect their average speed due to the changes in the particles' dwell times between successive jumps when the particle motion proceeds in short steps. Such an interaction leads to a speed-up of up to a factor of 60 compared with the velocity of an isolated thermal ratchet motor. Moreover, $\mathrm{HI}$ induces a surrounding fluid flow that allows transport of suspended particles with a mean velocity comparable to that of particles driving the flow. The phenomena that we describe should be relevant and experimentally observable, for example, in the collective transport of colloidal particles moving under the action of a ratchet potential generated by an optical trap although to our knowledge, such experiments have not yet been attempted. We expect our results are also relevant for biological systems where the steppers are molecular motors pulling a cargo. In particular, the geometry studied is of particular interest for axonal transport $[9,10]$ where the processive motor KIF1A has been proposed as a good experimental realization of the two state ratchet model. More generally, we expect our results to be relevant whenever the heterogeneous dynamic described by the two state model is fulfilled. This is found, for example, of nonprocessive motors whose tails are embedded in a membrane-covered cargo. In this case the fluidlike membrane [11] will sustain the hydrodynamic coupling while the proximity of the cargo to the filament will enhance the binding rate of nonprocessive motors. Finally, we speculate that the strong velocity enhancement that we observe may play a role in the very fast cytoplasmic streaming that has been observed in some plant cells [8].

P.M. and I.P. acknowledge financial support from MINECO (Spain) and DURSI under projects FIS201122603 and 2009SGR-634, respectively. D. F. acknowledges support from ERC Advanced Grant 227758, Wolfson Merit Award 2007/R3 of the Royal Society of London, and EPSRC Programme Grant EP/I001352/1.

*Corresponding author. paolomalgaretti@ffn.ub.es

[1] W. B. Russel, D. A. Saville, and W.R. Schowalter, Colloidal Dispersions (Cambridge University Press, Cambridge, England, 1992).

[2] S. Martin, M. Reichert, H. Stark, and T. Gisler, Phys. Rev. Lett. 97, 248301 (2006).

[3] R. D. Leonardo, S. Keen, F. Ianni, J. Leach, M. J. Padgett, and G. Ruocco, Phys. Rev. E 78, 031406 (2008).

[4] A. Grimm and H. Stark, Soft Matter 7, 3219 (2011).

[5] D. Houtman, I. Pagonabarraga, C.P. Lowe, A. M. C. Emons, and E. Eiser, Europhys. Lett. 78, 18001 (2007).

[6] A. V. Arzola, K. Volke-Sepúlveda, and J. L. Mateos, Phys. Rev. Lett. 106, 168104 (2011).

[7] F. Julicher, A. Ajdari, and J. Prost, Rev. Mod. Phys. 69, 1269 (1997).

[8] R. E. Goldstein, I. Tuval, V. D. Meent, and J. W, Proc. Natl. Acad. Sci. U.S.A. 105, 3663 (2008).

[9] P. Greulich, A. Garai, K. Nishinari, A. Schadschneider, and D. Chowdhury, Phys. Rev. E 75, 041905 (2007).

[10] Y. Okada and N. Hirokawa, Science 283, 1152 (1999).

[11] O. Campas, K. B. Zeldovich, P. Jolimaitre, L. BourelBonnet, P. Bassereau, J. Prost, and B. Goud, Proc. Natl. Acad. Sci. U.S.A. 101, 17096 (2004).

[12] $p_{12}$ and $p_{21}$ are chosen to optimize the velocity of an isolated motor [7].

[13] C. P. Lowe, Europhys. Lett. 47, 145 (1999).

[14] R. Pelton, Adv. Colloid Interface Sci. 85, 1 (2000).

[15] C. Lutz, M. Reichert, H. Stark, and C. Bechinger, Europhys. Lett. 74, 719 (2006).

[16] A. Igarashi, S. Tsukamoto, and H. Goko, Phys. Rev. E 64, 051908 (2001).

[17] J. Brugues and J. Casademunt, Phys. Rev. Lett. 102, 118104 (2009).

[18] Two steppers belong to the same cluster if their distance along the filament is smaller than $3 R$. 